\title{
The oxidation ratio of LDL: A predictor for coronary artery disease
}

\author{
Huiling Huang ${ }^{1}$, Weiyi Mai ${ }^{1}$, Dan Liu, Yuantao Hao, Jun Tao and Yugang Dong * \\ Department of Cardiology, the First Affiliated Hospital of Sun Yat Sen University, Guangzhou, PR China
}

\begin{abstract}
Objective: Oxidized LDL cholesterol (ox-LDL-C) is considered to be a key factor of initiating and accelerating atherosclerosis (AS). The purpose of this study is to elucidate the sensitivity and specificity of ox-LDL and oxidation ratio of LDL in the diagnosis of coronary artery disease (CAD). For the first time, we investigated the ratio of ox-LDL to ALB(ox-LDL/ALB). Methods and results: Blood ox-LDL, total cholesterol (TC), high density lipoprotein cholesterol (HDL-C), low density lipoprotein cholesterol (LDL-C), triglyceride (TG) and albumin (ALB) were measured in patients with acute myocardial infarction (AMI, $n=$ 80 ), unstable angina pectoris (UAP, $n=80$ ), stable angina pectoris (SAP, $n=80$ ), normal control $(n=60)$, and dyslipidemia control $(n=60)$. Ox-LDL was measured by competitive ELISA. The level of ox-LDL and oxidation ratio of LDL(ox-LDL/TC, ox-LDL/HDL-C, ox-LDL/ LDL-C and ox-LDL/ALB) were significantly higher in each diseased group than controls $(P<0.001)$. In CAD group, ox-LDL and oxidation ratio of LDL in subjects complicated with hypertension (HT) and/or diabetes mellitus (DM) increased further $(P<0.001)$. Ox-LDL/ALB in the AMI group was 7 times higher than normal control group $(0.068 \pm$ 0.017 vs $0.009 \pm 0.007, P<0.001$ ). The area under the curve (AUC) of receiver operating characteristic curve (ROC curve) is a criterium to evaluate the accuracy of diagnosing a disease. The AUC of ROC curve of ox-LDL/TC, ox-LDL/HDL-C, ox-LDL, ox-LDL/ALB and ox-LDL/ LDL-C for diagnosing CAD were $0.975,0.975,0.966,0.966,0.957$ respectively $(P<0.001)$. When ox-LDL/TC $=0.175$, the sensitivity and specificity of diagnosing CAD were 0.917 and 0.925 , which were almost equal to each other, indicating that the rates of missed diagnosis and misdiagnosis for CAD were the lowest.

Conclusions: The level of ox-LDL and the ratio of ox-LDL/TC, ox-LDL/LDL-C, ox-LDL/HDL-C and ox-LDL/ALB are better biomarkers than TC, TG, HDL-C and LDL-C for discriminating between patients with coronary artery disease and healthy subjects. And patients who have a high ratio of ox-LDL /TC may have a higher risk for CAD.
\end{abstract}

Keywords: Coronary artery disease, atherosclerosis, oxidized low density lipoprotein, cholesterol

\section{Introduction}

Atherosclerosis (AS) is a disease related to a series of risk factors, such as aging, dyslipidemia, inflammation, metabolic, and genetic factors, and the interaction of these factors contribute to the development of the disease. Among those, hypercholesterolemia has attracted most attention [1-3].

* Corresponding author: Yugang Dong, Department of Cardiology, First Affiliated Hospital of Sun Yat Sen University, 58 Zhong Shan Road II, Guangzhou, 510080, PR China. Tel.: +86 2087755766 ext 8151; Fax: +86 2087755766 ext 8756; E-mail: yg.dong@ medmail.com.cn.

${ }^{1}$ Co-first authors of the paper.
Hypercholesterolemia clearly plays a dominant role in the process [4,5]. Atherosclerosis is characterized by the deposition and accumulation of cholesterol in large and medium-sized arteries. This deposition leads to a proliferation of certain inflammatory cells within the arterial wall, formation of athoma and plaques that gradually narrow the vessel lumen and impede blood flow. This process may be quite insidious, and lasting for decades until rupture of plaques, resulting in acute coronary syndrome $[6,7]$.

There have been numerous hypotheses trying to explain the correlation of risk factors with the development of atherosclerosis [8-10]. Among them, three distinct hypotheses have been actively studied: the "response-to-injury hypothesis", "response-toretention hypothesis", and "oxidative modification hy- 
pothesis". Although each hypothesis points to its own critical initiating event, there are some common features. For example, each involves a significant component of inflammation and each indicates LDL as a central element. The oxidative modification hypothesis has been studied intensively. Without a doubt, there is certainly an oxidation of lipoprotein in vivo. OxLDL and the other oxidated lipid as well as its degradation product induce the development of atherosclerosis by various mechanisms, proinflammatory, proimmune reaction and cytotoxicity.

There are some reports showing that blood ox-LDL was elevated in patients with coronary artery disease, suggesting that ox-LDL may be a marker for atherosclerosis [11-13]. Moreover, patients with acute coronary syndromes, such as acute myocardial infarction and unstable angina, have been reported to have higher plasma levels of oxidized LDL than patients with stable angina [15], suggesting that plasma ox-LDL levels correlate with the severity of coronary stenoses. Fredrikson GN et al. reported that high plasma ox-LDL/TC ratio is at increased risk for development of AMI, but they had not formulated the concrete threshold. As the variation of ox-LDL in the circulation is big, it is difficult to work out a specific figure to evaluate the risk for CAD according to it so far. Although Fredrikson GN et al found that high plasma ox-LDL/TC is at increased risk for development of AMI, they had not compared the sensitivity and specificity with other biomarkers as TC, HDL-C and LDL-C. For this reason, this research aims directly at not only the total level of oxidized LDL in the circulation but also the oxidation ratio of $\mathrm{LDL}$ (ox-LDL/TC, ox-LDL/HDL-C, ox-LDL/ LDL-C and ox-LDL/ALB) in the diagnosis of CAD. And it compares the sensitivity and specificity of TC, HDL-C and LDL-C in the diagnosing of CAD.

It was reported that developing lesions had decreased ratio of ALB to LDL in the plasma [7]. In this study, for the first time, we investigated the ratio of ox-LDL to ALB (ox-LDL/ALB), as to elucidate that whether it is a better biomarker in discriminating between patients with coronary artery disease and healthy subjects.

\section{Methods}

\subsection{Study population}

Three hundred and sixty individuals were studied: 80 patients with acute myocardial infarction (AMI), 80 patients with unstable angina (UAP), 80 patients with stable angina (SAP). 120 control people were divided into normal control $(n=60)$ and dyslipidemia control ( $n=60)$. The normal control were healthy subjects with normal blood lipid, the dyslipidemia control were individuals without evidence of CAD, stroke, hypertension, diabetes mellitus, and whose LDL-C above $3.62 \mathrm{mmol} / \mathrm{l}$ or TC above $5.70 \mathrm{mmol} / \mathrm{l}$. Individuals whose creatinin $>115 \mu \mathrm{mol} / \mathrm{l}$, or glutamate-pyruvate transaminase $(\mathrm{GPT})>2$ times the upper normal limit, or has an evidence of stroke were excluded from this study. Considering that statins can inhibit oxidative stress, anyone who treated with statins three months within sampling was excluded from the study. Population characters were showed on Table 1A and 1B.

The diagnosis of AMI was based on a history of prolonged ischemic chest pain, characteristic ECG changes, and elevated blood serum markers. UAP was defined as new-onset of angina within 2 months after a previous bout, or angina with a progressive crescendo pattern, or with the anginal episodes increasing in frequency and/or duration, or angina occurred at rest, the chest pain lasted less than 30 minutes. SAP was diagnosed as chest pain typical of cardiac ischemia on exertion. The diagnosis of UAP and SAP must be confirmed by a document of ischemia, electrocardiogram or angiogram.

\subsection{Laboratory analysis}

Overnight fasting blood samples were drawn for measurement of ox-LDL, total cholesterol (TC), triglycerides (TG), HDL cholesterol (HDL-C), LDL cholesterol (LDL-C), fasting plasma glucose and albumin. TC and TG were measured by enzymic method, HDL-C and LDL-C were measured by homogeneous method, and ALB was measured by bromocresol green (BCG) method. Ox-LDL was measured by competitive, and mAb4E6 antibody, developed by Holvoet et al. [12]. In brief, $100 \mu$ l plasma was added in a microtiter well coated with goat anti-human ox-LDL antibody of a plate, (Mercodia, Cat: TE212). The plate was put on a shaker and shaking gently for five minutes at room temperature (RT). Then it was put on a bench and incubated at RT for one hour without shaking. Ox-LDL in the plasma would bind to the coated antibody. After sucking out the supernatant in the wells, the wells were washed with $1 \mathrm{X}$ washing buffer (offered with the kit) for six times. After the final wash, the plate was inverted and tapped firmly against absorbent paper. After these procedures, the composition that had not binded to the coated antibody was washed 
Table 1A

Baseline character of each group. Data are expressed as mean \pm SD or as percent

\begin{tabular}{|c|c|c|c|c|c|c|c|}
\hline & $\begin{array}{c}\text { Sex } \\
(\text { male \%) }\end{array}$ & $\begin{array}{c}\text { Age } \\
\text { (years) }\end{array}$ & $\begin{array}{c}\text { BMI } \\
\left(\mathrm{Kg} / \mathrm{m}^{2}\right)\end{array}$ & $\begin{array}{c}\text { Never } \\
\text { smokers }(\%)\end{array}$ & $\begin{array}{c}\text { Former } \\
\text { smokers }(\%)\end{array}$ & $\begin{array}{c}\text { Current } \\
\text { smokers }(\%)\end{array}$ & $\begin{array}{c}\mathrm{Cr} \\
(\mu \mathrm{mol} / \mathrm{l})\end{array}$ \\
\hline $\operatorname{SAP}(n=80)$ & 56.3 & $63.4 \pm 8.7$ & $23.90 \pm 3.46^{+\phi}$ & 47.5 & 27.5 & 25.0 & $85.74 \pm 3.15$ \\
\hline $\operatorname{UAP}(n=80)$ & 53.8 & $63.3 \pm 9.6$ & $23.23 \pm 2.65^{+}$ & 51.3 & 27.5 & 21.3 & $94.11 \pm 7.27$ \\
\hline AMI $(n=80)$ & 77.5 & $64.1 \pm 10.0$ & $24.47 \pm 2.17^{*}$ & 47.5 & 28.8 & 23.8 & $107.52 \pm 8.16$ \\
\hline Normal control $(n=60)$ & 53.3 & $63.4 \pm 6.5$ & $22.91 \pm 3.60$ & 53.3 & 23.3 & 23.3 & $88.43 \pm 3.60$ \\
\hline Dyslipidemia control $(n=60)$ & 53.3 & $62.8 \pm 4.2$ & $24.46 \pm 3.29^{*}$ & 55.0 & 21.7 & 23.3 & $82.40 \pm 4.60$ \\
\hline Sig & 0.05 & NS & 0.05 & NS & NS & NS & NS \\
\hline
\end{tabular}

${ }^{*} p<0.001$ vs group Normal control. $\oint_{p}<0.001$ vs group UAP. ${ }^{\triangle} p<0.001$ vs group Dyslipidemia control. ${ }^{+} p<0.001$ vs group AMI.

Table 1B

Lipid profile in each group

\begin{tabular}{|c|c|c|c|c|c|c|c|}
\hline & $\begin{array}{c}\text { FPG } \\
(\mathrm{mmol} / \mathrm{l})\end{array}$ & $\begin{array}{c}\mathrm{TC} \\
(\mathrm{mmol} / \mathrm{l})\end{array}$ & $\begin{array}{c}\mathrm{TG} \\
(\mathrm{mmol} / \mathrm{l})\end{array}$ & $\begin{array}{l}\text { HDL-C } \\
(\mathrm{mmol} / \mathrm{l})\end{array}$ & $\begin{array}{l}\text { LDL-C } \\
(\mathrm{mmol} / \mathrm{l})\end{array}$ & $\begin{array}{r}\text { ALB } \\
(\mathrm{g} / 1)\end{array}$ & LDL-C/TC \\
\hline $\operatorname{SAP}(n=80)$ & $5.95 \pm 1.88^{*} \oint$ & $4.87 \pm 0.77^{*}$ & $1.58 \pm 0.81$ & $1.05 \pm 0.24^{*}$ & $3.03 \pm 0.71^{*+}$ & $42.49 \pm 3.28^{+}$ & $0.63 \pm 0.09$ \\
\hline $\operatorname{UAP}(n=80)$ & $5.24 \pm 1.28^{*}$ & $4.89 \pm 0.73^{*}$ & $1.73 \pm 1.14$ & $1.07 \pm 0.26^{*}$ & $3.12 \pm 0.66^{*+}$ & $41.72 \pm 2.90^{+}$ & $0.62 \pm 0.10$ \\
\hline AMI $(n=80)$ & $6.20 \pm 2.22 * \oint$ & $5.06 \pm 0.79^{*}$ & $1.50 \pm 0.71$ & $0.98 \pm 0.29 *$ & $3.43 \pm 0.70^{*}$ & $40.43 \pm 2.89$ & $0.65 \pm 0.10^{*}$ \\
\hline $\begin{array}{l}\text { Normal control } \\
\quad(n=60)\end{array}$ & $4.58 \pm 0.66$ & $4.56 \pm 0.75$ & $1.66 \pm 1.29$ & $1.20 \pm 0.28$ & $2.68 \pm 0.51$ & $42.82 \pm 3.83^{+}$ & $0.59 \pm 0.09$ \\
\hline $\begin{array}{l}\text { Dyslipidemia control } \\
\qquad(n=60)\end{array}$ & $5.07 \pm 1.04$ & $6.43 \pm 0.84^{*}$ & $1.68 \pm 0.76$ & $1.06 \pm 0.12^{*}$ & $4.25 \pm 0.54^{*}$ & $41.54 \pm 3.40^{+}$ & $0.66 \pm 0.05^{*}$ \\
\hline Sig & 0.000 & 0.000 & NS & 0.000 & 0.000 & 0.000 & 0.001 \\
\hline
\end{tabular}

${ }^{*} p<0.001$ vs group Normal control. $\Phi_{p}<0.001$ vs group UAP. ${ }^{\triangle} p<0.001$ vs group Dyslipidemia control. ${ }^{+} p<0.001$ vs group AMI. ALB: albumin.. HDL-C: high density lipoprotein cholesterol. TC: total cholesterol. FPG: fasting plasma glucose. LDL-C: low density lipoprotein cholesterol. TG: triglyceride.

off. $100 \mu \mathrm{l}$ biotin-conjugated m4E6 antibody (1X, CXAB032) was added in each well, and shaken gently for five minutes, then incubated at RT for one hour. The biotin-conjugated m4E6 antibody would bind to the ox-LDL captured by goat anti-human antibody. Supernatant was removed, and wells were washed with washing buffer for six times. The unbinded composition was washed off. $100 \mu$ l HRP-conjugated streptavidin (diluted according to the manual) was added in each well, incubated at RT for one hour on a shaker and shaken gently. And then wells were washed with washing buffer for six times. After the final wash, the plate was inverted and tapped firmly against absorbent paper. $100 \mu 1$ 3,3',5,5' - tetramethylbenzidine(TMB) was added in each well and incubated at RT for twenty minutes without shaking. Then $100 \mu \mathrm{l}$ stop solution $(0.5 \mathrm{M}$ $\mathrm{H}_{2} \mathrm{SO}_{4}$ ) was added in each well and shaken gently for thirty seconds to stop the reaction. OD was read by a spectrophotometer (Stat Fax-2100£<Awareness, UAS). All samples were detected triplicated. The coefficients of variation for intraassay and interassay were $0.9 \%$ to $2.2 \%$ and $1.3 \%$ to $3.8 \%$, respectively.

\subsection{Statistics}

SPSS 13.0 was used for the statistical analyses. ANOVA and rank test were applied for significance tests. Chi-square test was applied to compare the difference of sex and smoking in each group. Receiveroperating characteristic curves were used to evaluate the diagnostic accuracy of the candidate markers. Sensitivity was plotted on the $\mathrm{Y}$ axis and the proportion of false positives (1-specificity) on the $\mathrm{X}$ axis. AUC is considered to be the value of accuracy for diagnosing a disease, the closer to 1 , the more accurate the diagnosis. It will lose the diagnostic value when it is less than 0.5 .

\section{Results}

\section{1. $O x-L D L$, ox-LDL/TC, ox- $L D L / L D L-C$, ox-LDL/HDL-C and ox-LDL/ALB in each group}

Ox-LDL, ox-LDL/TC, ox-LDL/LDL-C, ox-LDL/ HDL-C and ox-LDL/ALB in each group are shown on Table 1C and Fig. 1. Mean value of ox-LDL is significantly higher in the AMI group than in the UAP or SAP groups, or in controls, similar to the reports before [15], and the ratio ox-LDL/TC, ox-LDL/LDL$\mathrm{C}$, ox-LDL/HDL-C and ox-LDL/ALB was significantly higher in the CAD groups than in the two control groups $(P<0.001)$. 
Table 1C

Serum oxidized LDL and oxidation ratio of LDL in each group

\begin{tabular}{|c|c|c|c|c|c|}
\hline & $\begin{array}{l}\text { Ox-LDL } \\
(\mathrm{mmol} / \mathrm{l}) \\
\end{array}$ & Ox-LDL/TC & Ox-LDL/HDL-C & Ox-LDL/LDL-C & $\begin{array}{c}\text { ox-LDL/ALB } \\
(\mathrm{mmol} / \mathrm{l})\end{array}$ \\
\hline $\operatorname{SAP}(n=80)$ & $1.45 \pm 0.75^{*} \phi^{+}$ & $0.30 \pm 0.14^{*} \phi^{+}$ & $1.47 \pm 0.90 * \oint+$ & $0.48 \pm 0.21 * \oint+$ & $0.03 \pm 0.02^{*}$ \\
\hline $\operatorname{UAP}(n=80)$ & $1.94 \pm 0.80^{*+}$ & $0.40 \pm 0.16^{*+}$ & $1.95 \pm 1.08^{*+}$ & $0.63 \pm 0.23^{*+}$ & $0.05 \pm 0.02^{*}$ \\
\hline AMI $(n=80)$ & $2.70 \pm 0.65^{*}$ & $0.54 \pm 0.11^{*}$ & $3.15 \pm 1.52^{*}$ & $0.80 \pm 0.16^{*}$ & $0.07 \pm 0.02^{*}$ \\
\hline Normal control $(n=60)$ & $0.43 \pm 0.26$ & $0.10 \pm 0.06$ & $0.38 \pm 0.26$ & $0.17 \pm 0.11$ & $0.01 \pm 0.01$ \\
\hline Dyslipidemia control $(n=60)$ & $0.55 \pm 0.26$ & $0.09 \pm 0.04$ & $0.53 \pm 0.26$ & $0.13 \pm 0.06$ & $0.01 \pm 0.01^{*}$ \\
\hline Sig & 0.000 & 0.000 & 0.000 & 0.000 & 0.000 \\
\hline
\end{tabular}

${ }^{*} p<0.001$ vs group Normal control. ${ }^{\oint} p<0.001$ vs group UAP. ${ }^{\triangle} p<0.001$ vs group Dyslipidemia control. ${ }^{+} p<0.001$ vs group AMI. ALB: albumin.. HDL-C: high density lipoprotein cholesterol. LDL-C: low density lipoprotein cholesterol. Ox-LDL: oxidized low density lipoprotein. TC: total cholesterol.
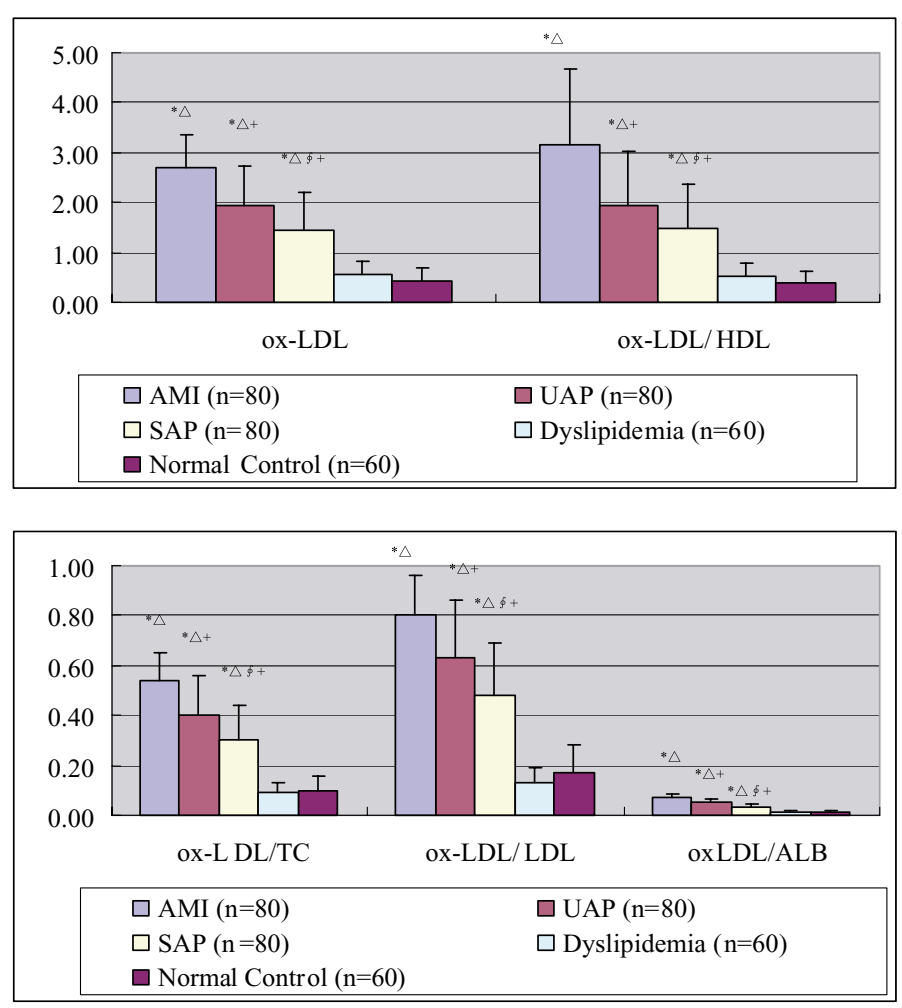

\footnotetext{
*: $\mathrm{p}<0.001$ vs group Normal control.

$\triangle: \mathrm{p}<0.001$ vs group Dyslipidemia control.

$\phi: \mathrm{p}<0.001$ vs group UAP

$+: \mathrm{p}<0.001$ vs group AMI
}

Fig. 1. Serum oxidized LDL (a), oxidized LDL/total cholesterol (b), oxidized LDL/LDL cholesterol (c), oxidized LDL/HDL cholesterol ratio (d) and oxidized LDL/ALB ratio (e) in each group.

\section{2. $O x-L D L$ and oxidation ratio of $L D L$ in $C A D$ patients complicated with diabetes mellitus $(D M)$ and hypertension $(H T)$}

As to evaluate the impact of hypertension and diabetes on oxidation of LDL, we further categorized patients with coronary artery disease (defined as stable angina, or unstable angina, or acute myocardial infarction, 240 cases all together) into group of coronary artery disease alone (CAD), coronary artery disease with hypertension $(\mathrm{CAD}+\mathrm{HT})$, coronary artery disease with diabetes $(\mathrm{CAD}+\mathrm{DM})$, and coronary artery disease with hypertension and diabetes $(\mathrm{CAD}+\mathrm{HT}+\mathrm{DM})$ based on their illness situation. Data is shown in Fig. 2. The study further found that ox-LDL, ox-LDL/TC, oxLDL/LDL-C, ox-LDL/HDL-C and ox-LDL/ALB were higher in group $\mathrm{CAD}+\mathrm{HT}$ and $\mathrm{CAD}+\mathrm{DM}$, while highest in group $\mathrm{CAD}+\mathrm{HT}+\mathrm{DM}$, indicating that hyperten- 

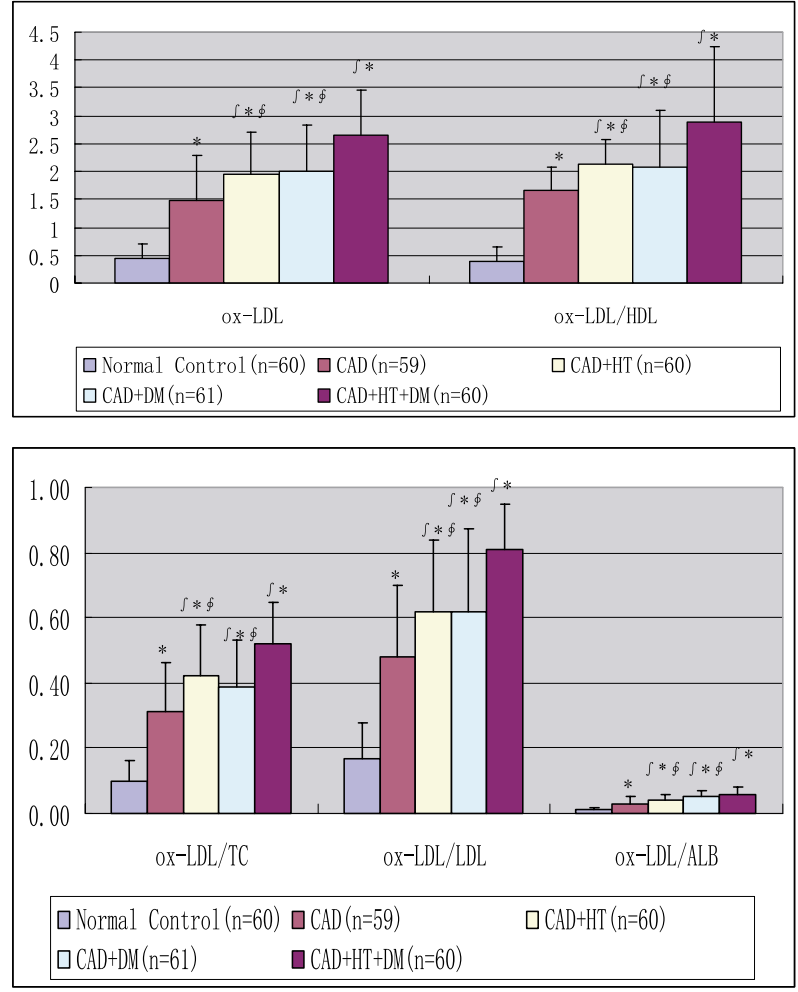

$\int: p<0.001$ vs group CAD.

*: p $\langle 0.001$ vs group Normal Control.

Fig. 2. Serum oxidized LDL, oxidized LDL/total cholesterol, oxidized LDL/LDL cholesterol, oxidized LDL/HDL cholesterol ratio and ALB/ oxidized LDL ratio in subjects with CAD, CAD + HT, CAD+DM and CAD+HT+DM.

sion and diabetes may accelerate the oxidation of LDL.

\subsection{Receiver-operating characteristic analyses}

The predictive value of ox-LDL, ox-LDL/TC, oxLDL/HDL-C, ox-LDL/LDL-C and ox-LDL/ALB for $\mathrm{CAD}$, the receiver-operating characteristic curve (ROC curve), and the area under the curve(AUC) for diagnosing CAD are shown in Table 2 and Fig. 3. The AUC of ox-LDL, ox-LDL/TC, ox-LDL/HDL-C, ox-LDL/LDL$\mathrm{C}$ and ox-LDL/ALB were more than $0.50(P<0.001)$. When ox-LDL/TC ratio $=0.175$, the sensitivity is close to the specificity, which are 0.917 and 0.925 respectively. Compared to ox-LDL, the sensitivity and specificity are larger in ox-LDL/TC, ox-LDL/LDL-C and ox-LDL/ALB (Table 2). Furthermore, the variations of ox-LDL/TC and ox-LDL/ALB were the minimum $(P<0.05$, Table 3$)$. According to the principle of statistics, when the sensitivity is close to the specificity, the value of accuracy for diagnosing a disease is the best. It suggested that when plasma ox-LDL/TC ratio is over 0.175 , the rate of missed diagnosis and misdiagnosis for CAD were the lowest.

\section{Discussion and conclusion}

There is increasing evidence that markers of inflammatory activity are increased in CAD patients [15]. Clinical studies have suggested that high levels of inflammatory markers are associated with an increased risk for development of CAD [21], because inflammation of the arterial intima is considered as one of the major characteristics of atherosclerosis. Accumulation, aggregation and oxidative modification of LDL are regarded to play a pivotal role in activation of this inflammation [14]. Endothelial cells, smooth muscle cells and macrophages may release the oxygen free radicals, and then oxidize LDL to ox-LDL. Some studies have shown that the level of ox-LDL may indicate the severity of pathological inflammatory response [22,23]. The recent development of ELISA for oxidized LDL using monoclonal antibodies has made it possible to identify oxidized LDL also in the circulation [12]. Increased levels of oxidized LDL have been demonstrated in patients with coronary artery disease $£$ <and it suggests that 
Table 2

Area under the ROC curve, sensitivity and 1-specificity of nine biomarkers

\begin{tabular}{lccccccccc}
\hline & TC & HDL-C & LDL-C & LDL/TC & Ox-LDL & Ox-LDL/TC & Ox-LDL/LDL-C & Ox-LDL/HDL-C & Ox-LDL/ALB \\
\hline AUC & 0.365 & 0.375 & 0.410 & 0.517 & 0.966 & 0.975 & 0.975 & 0.957 & 0.966 \\
Threshold & 5.035 & 1.025 & 3.345 & 0.623 & 0.860 & 0.175 & 0.275 & 0.775 & 0.021 \\
Sensitivity & 0.425 & 0.450 & 0.425 & 0.575 & 0.908 & 0.917 & 0.925 & 0.879 & 0.904 \\
1-Specificity & 0.592 & 0.675 & 0.675 & 0.592 & 0.100 & 0.075 & 0.075 & 0.158 & 0.083 \\
\hline
\end{tabular}

\section{ROC Curve}

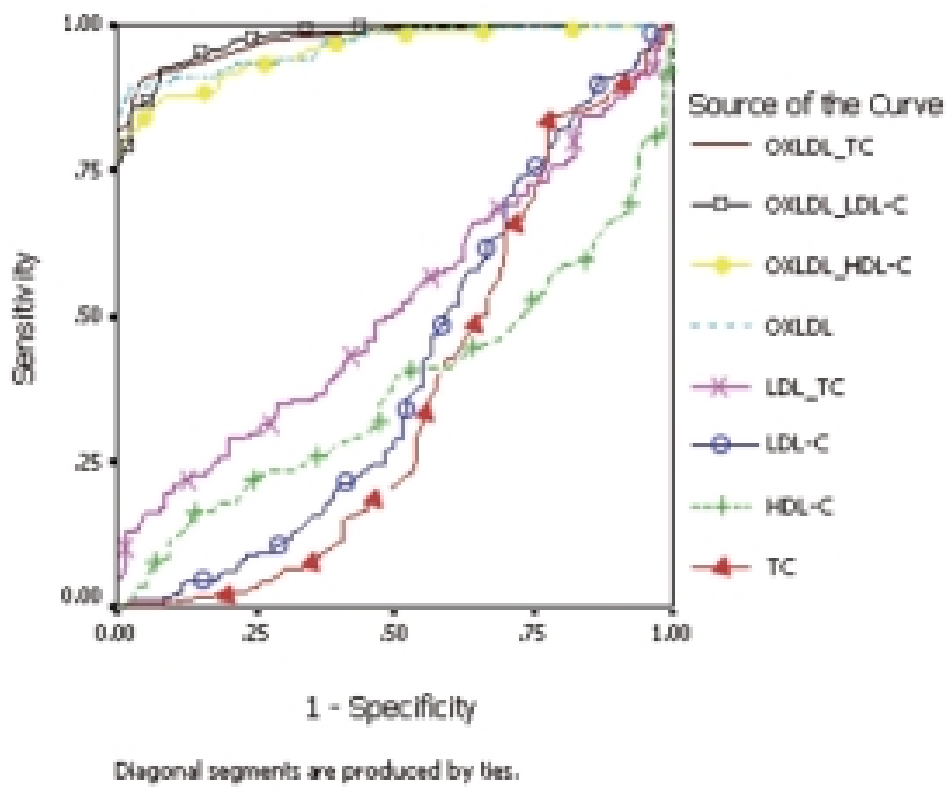

Fig. 3. Receiver-operating characteristic curves for lipid and lipoprotein biomarkers. HDL-C $=$ HDL cholesterol; LDL-C $=$ LDL cholesterol; OXLDL $=$ oxidized LDL; TC $=$ Total cholesterol.

the plasma level of oxidized LDL may be a marker of CAD.

Plasma ox-LDL levels may change in individuals during different stages of a disease. Several reports have shown that ox-LDL levels increase in the acute phase immediately after AMI, or percutaneous transluminal coronary angiography(PTCA) intervention. One possible explanation for the temporal increase is that ox-LDL can be released from ruptured plaques into the circulation at the time of occurrence of infarction [20].

In this study, we found that the level of ox-LDL in the plasma was more than 6 times higher in patients with AMI than in control subjects. This strongly suggests that ox-LDL in plasma could serve as a marker for cardiovascular events.

Besides, the plasma ox-LDL/TC, ox-LDL/LDL-C, and ox-LDL/HDL-C ratio were significantly higher in CAD groups than controls. The finding is consistant with the previous studies [12,15]. Fredrikson et al. [21] reported that high plasma ox-LDL/TC ratio is at in- creased risk for development of AMI. Ehara et al. [15] reported that patients with stable angina pectoris had $50 \%$ higher oxidized LDL/LDL protein than controls. These findings indicate that the risk for CAD is associated with the relative degree of LDL oxidation rather than with the total level of oxidized LDL in vivo.

However, these two studies had not compared the sensitivity and specificity of ox-LDL/TC or oxLDL/LDL-C with that of other biomarkers as TC, LDL$\mathrm{C}$ in the diagnosing of CAD. So they did not give us an idea of how good they were in the diagnosing of the disease.

It was reported that developing lesions had decreased ratio of ALB to LDL in the plasma [7]. Relative decreases in albumin may promote the availability of transition metal ions via decreasing metal binding, or lowering the concentration of sacrificial thiols to scavenge $2 e$-oxidants [7]. In this study, for the first time, we investigated the ratio of ox-LDL to ALB (ox-LDL/ALB), the result suggests that ox-LDL/ALB in CAD group 
Table 3

The comparison of the variation of ox-LDL, ox-LDL/TC, ox-LDL/HDL-C and ox-LDL/LDL-C

\begin{tabular}{lcccccc}
\hline Biomarker & group & Mean & $\begin{array}{c}\text { Std. } \\
\text { deviation }\end{array}$ & Minimum & Maximum & $\begin{array}{c}95 \% \text { confidence } \\
\text { interval }\end{array}$ \\
\hline ox-LDL & 0 & 0.490 & 0.267 & 0.040 & 1.072 & $0.442 \sim 0.539$ \\
ox-LDL/TC & 1 & 2.028 & 0.896 & 0.213 & 4.281 & $1.914 \sim 2.142$ \\
& 0 & 0.091 & 0.051 & 0.011 & 0.224 & $0.083 \sim 0.101$ \\
ox-LDL/LDL-C & 1 & 0.408 & 0.166 & 0.045 & 0.783 & $0.388 \sim 0.430$ \\
ox-LDL/HDL-C & 0 & 0.149 & 0.089 & 0.011 & 0.434 & $0.133 \sim 0.166$ \\
ox-LDL/ALB & 1 & 0.635 & 0.241 & 0.072 & 0.922 & $0.604 \sim 0.666$ \\
& 0 & 0.452 & 0.267 & 0.043 & 1.238 & $0.404 \sim 0.501$ \\
& 1 & 2.191 & 1.385 & 0.172 & 8.521 & $2.015 \sim 2.367$ \\
& 0.011 & 0.007 & 0.002 & 0.034 & $0.010 \sim 0.012$ \\
& 1 & 0.049 & 0.023 & 0.004 & 0.114 & $0.046 \sim 0.053$ \\
\hline
\end{tabular}

0 : subjects without coronary artery disease. 1 : subjects with coronary artery disease.

was higher than the normal control group, especially the AMI group. The ratio in the CAD group is 7 times more than the normal control group. It suggests that the patients having a high ratio of ox-LDL/ALB may have higher risk for AMI. It may be a candidate marker to evaluate the risk for AMI, or the severity of CAD (data shown in Table 1C and Fig. 1).

There were two controls in the study. Individuals with hypertension or diabetes were excluded from these two controls. Hypertension and diabetes are risk factors of CAD. As to evaluate the contribution to these factors to oxidation of LDL, we categorized the patients with CAD into CAD only, CAD+HT, CAD+DM and $\mathrm{CAD}+\mathrm{HT}+\mathrm{DM}$ based on the disease situation. The study further found that ox-LDL, ox-LDL/TC, oxLDL/LDL-C, ox-LDL/HDL-C and ox-LDL/ALB ratio were higher in group $\mathrm{CAD}+\mathrm{HT}$ and $\mathrm{CAD}+\mathrm{DM}$ than in controls, while highest in group $\mathrm{CAD}+\mathrm{HT}+\mathrm{DM}$. It suggested that oxidative modification of LDL played a pivotal role in activation of this inflammation. Hypertension and diabetes may accelerate the oxidation of LDL.

It was said that oxidative stress was a common pathophysiologic pathway of insulin resistance (IR), diabetes and coronary artery disease. Individuals with DM are at increased risk for developing CAD as well as for mortality from CAD and other causes [16]. Because DM is associated with high risk for atherosclerotic disease, a strong oxidation of LDL may be involved in the process. Hyperglycemia, hyperinsulinemia, hypertension and cigarette smoking can induce oxidative stress, release of oxygen free radicals from inflammatory cells, and then promote the oxidation of LDL to ox-LDL. Glucose can also directly react with LDL phospholipids and apolipoprotein $\mathrm{B}$ (apoB) lysine groups to form the advanced glycation end products (AGEs) that facilitate lipid peroxidation [17].
In this study, we compared the diagnostic accuracy of 9 different lipid or lipoprotein biomarkers that have been suggested to identify patients with an increased risk of CAD. Diagnostic accuracy was determined by measuring the area under the curve of the receiveroperating characteristic curve, which is the most common method for quantifying and comparing the accuracies of different diagnostic tests. The present observations suggest that oxidized LDL, especially when used in combination with TC or ALB, are better biomarkers than popular lipid profile (TC, LDL-C) for discriminating between patients with CAD and healthy subjects. And the patients who have a ratio of ox-LDL /TC over 0.175 , or ox-LDL /ALB over 0.021 may have a higher risk for CAD.

So far, there are 3 other studies in which oxidized LDL and traditional lipid or lipoprotein biomarkers were measured in patients with CAD and compared with non-CAD subjects [12,18,19]. However, our study is different from them in the following. First, there were two controls in the study. Individuals who have hypertension or diabetes were excluded from these two controls. Ox-LDL, ox-LDL/TC, ox-LDL/LDL-C, ox-LDL/HDL-C and ox-LDL/ALB ratio were higher in CAD groups than that in controls. Second, none of the other studies have evaluated the diagnostic accuracy of ox-LDL/TC, ox-LDL/LDL-C, ox- LDL/ HDL-C and ox-LDL/ALB for CAD in a comparable study. Ehara et al. [15] found that ox-LDL levels show a significant positive correlation with the severity of acute coronary syndromes. Consistent with this finding, we found that ox-LDL/TC was highest in the AMI group, then the UAP group, and then with SAP group. It indicated that as the increasing of ox-LDL/TC, the severity of CAD was increasing. Our study suggested that they are all better indicators of CAD than TC and LDL-C. We believe that ox-LDL/TC ratio or ox-LDL/ALB is a possible indicator of increased risk for CAD. Patients who 
have a high ratio of ox-LDL/TC or ox-LDL/ALB, may have higher risk for CAD. Third, for the first time, we investigated the value of ox-LDL/ALB ratio in the diagnosis of CAD. We found that the ratio of ox-LDL/ALB in the AMI group is 7 times more than normal control, indicating that patients having high ratio of oxLDL/ALB may have higher risk for AMI. It may be a candidate marker to evaluate the risk for AMI, or the severity of CAD.

In conclusion, this study found that the levels of oxLDL, ox-LDL/TC, ox-LDL/ HDL-C, ox-LDL/ LDL-C and ox-LDL/ALB were significantly higher in CAD patients, subjects with hypertension and/or diabetes $(P<$ 0.001). Ox-LDL/ALB in the AMI group is 7 times more than normal control. This study suggests that oxLDL, ox-LDL/TC, ox-LDL/LDL-C, ox-LDL/HDL-C and ox-LDL/ALB are better biomarkers for discriminating between patients with coronary artery disease and healthy subjects. And when the ratio of ox-LDL /TC is over 0.175 , the rate of missed diagnosis and misdiagnosis for CAD were the lowest.

\section{Acknowledgements}

We sincerely thank Prof ZhiJie Zeng for his assistance of measuring ox-LDL. We also thank Prof ZeFang Ren for his assistance with statistics and helpful suggestion.

The project is supported by Scientific Research Fund of Guangdong (No: 2005B30601019).

\section{References}

[1] S.C. Smith Jr., J.L. Anderson, R.O. Cannon III, Y.Y. Fadl, W. Koenig, P. Libby, S.E. Lipshultz, G.A. Mensah, P.M. Ridker and R. Rosenson, CDC/AHA workshop on markers of inflammation and cardiovascular disease: application to clinical and public health practice: report from the clinical practice discussion group, Circulation 110 (2004), 550-553.

[2] B. Philip, K. John, N. Alistair and H. Richard, Future Forum Editorial Board. High density lipoproteins (HDLs) and atherosclerosis; the unanswered questions, Atherosclerosis 168 (2003), 195-211.

[3] M. Anselmi, U. Garbin, P. Agostoni et al., Plasma levels of oxidized-low-density lipoproteins are higher in patients with unstable angina and correlated with angiographic coronary complex plaques, Atherosclerosis 185 (2006), 114-120.

[4] J. Galle, T. Hansen-Hagge, C. Wanner and S. Seibold, Impact of oxidized low density lipoprotein on vascular cells, Atherosclerosis 185 (2006), 219-226.

[5] P. Subramaniam, B. Constanze, S. Baohai et al., Human Atherosclerotic Intima and Blood of Patients with Established Coronary Artery Disease Contain High Density Lipoprotein Damaged by Reactive Nitrogen Species, J Biol Chem 279 (2004), 42977-42983.
[6] R.J. Esper, From risk factors of arterial disease to atheroma plaque detection of the high-risk plaque, Rev Fac Cien Med Univ Nac Cordoba 61(Suppl 1) (2004), 39-52.

[7] S. Roland, F. John and J.R. Keaney, Role of Oxidative Modifications in Atherosclerosis, Physiol Rev 84 (2004), 1381-1478.

[8] K. Skalen, M. Gustafsson, E.K. Rydberg, L.M. Hulten, O. Wiklund, T.L. Innerarity and J. Borén, Subendothelial retention of atherogenic lipoproteins in early atherosclerosis, $\mathrm{Na}$ ture 417 (2002), 750-754.

[9] P. Libby, Inflammation in atherosclerosis, Nature 420 (2002), 868-874.

[10] C. Flood, M. Gustafsson, P.E. Richardson, S.C. Harvey, J.P. Segrest and J. Borén, Identification of the proteoglycan binding site in apolipoprotein B48, J Biol Chem 277 (2002), 32228-32233.

[11] P. Holvoet, J. Vanhaecke, S. Janssens, V.D.W. Frans and C. Désiré, Oxidized LDL and malondialdehyde-modified LDL in patients with acute coronary syndromes and stable coronary artery disease, Circulation 98 (1998), 1487-1494.

[12] S. Toru and K. Hiroaki, Diagnostic implications of circulating oxidized low density lipoprotein levels as a biochemical risk marker of coronary artery disease, Clinical Biochemistry $\mathbf{3 5}$ (2002), 347-353.

[13] S. Kazunori and M. Hiroshi, Circulating oxidized low-density lipoprotein is an independent predictor for cardiac event in patients with coronary artery disease, Atherosclerosis 174 (2004), 343-347.

[14] C.K. Glass and J.L. Witztum, Atherosclerosis: the road ahead, Cell 104 (2001), 503-516.

[15] S. Ehara, M. Ueda, T. Naruko, K. Haze, A. Itoh, M. Otsuka, R. Komatsu, T. Matsuo, H. Itabe, T. Takano, Y. Tsukamoto, M. Yoshiyama, K. Takeuchi, J. Yoshikawa and A.E. Becker, Elevated levels of oxidized low density lipoprotein show a positive relationship with the severity of acute coronary syndromes, Circulation 103 (2001), 1955-1960.

[16] B. Isomaa, P. Almgren, T. Tuomi, B. Forsen, K. Lahti, M. Nissen, M.R. Taskinen and L. Groop, Cardiovascular morbidity and mortality associated with the metabolic syndrome, Diabetes Care 24 (2001), 683-689.

[17] P. Holvoet, B.K. Stephen, P.T. Russell, M. Ann, M.R. Susan, B. Javed, G. Bret and B.H. Tamara, The Metabolic Syndrome, Circulating Oxidized LDL, and Risk of Myocardial Infarction in Well-Functioning Elderly People in the Health, Aging, and Body Composition Cohort, Diabetes 53 (2004), 1068-1073.

[18] S. Ehara, M. Ueda, K. Haze, T. Naruko, A. Itoh, M. Otsuka, R. Komatsu, T. Matsuo, H. Itabe, T. Takano, Y. Tsukamoto, M. Yoshiyama, K. Takeuchi, J. Yoshikawa and A.E. Becher, Elevated Levels of Oxidized Low Density Lipoprotein Show a Positive Relationship With the Severity of Acute Coronary Syndromes, Circulation 17 (2001), 1955-1960.

[19] N. Johnston, T. Jernberg, B. Lagerqvist, A. Siegbahn and L. Wallentin, Improved Identification of Patients With Coronary Artery Disease by the Use of New Lipid and Lipoprotein Biomarkers, Am J Cardiol 97 (2006), 640-645.

[20] H. Itabe and M. Ueda, Measurement of plasma oxidized Low-Density Lipoprotein and its Clinical Implications, $J$ Atheroscler Thromb 14 (2007), 1-11.

[21] G.N. Fredrikson, B. Hedblad, G. Berglund and J. Nilsson, Plasma oxidized LDL: a predictor for acute myocardial infarction? J Internal Medicine 253 (2003), 425-429.

[22] P. Holvoet, T.B. Harris, R.P. Tracy, P. Verhamme, A.B. Newman, S.M. Rubin, E.M. Simonsick, L.H. Colbert and S.B. Kritchevsky, Association of High Coronary Heart Disease Risk Status With Circulating Oxidized LDL in the Well- 
Functioning Elderly. Findings From the Health, Aging, and Body Composition Study, Arterioscler Thromb Vasc Biol $\mathbf{2 3}$ (2003), 1444-1448.

[23] P. Kampusa, J. Kals, T. Ristimaë, P. Muda, K. Ulst, K. Zilmer, R.M. Salonen, T.P. Tuomainen, R. Teesalu and M. Zilmer,
Augmentation index and carotid intima-media thickness are differently related to age, C-reactive protein and oxidized lowdensity lipoprotein, Journal of Hypertension 25 (2007), 819825 . 


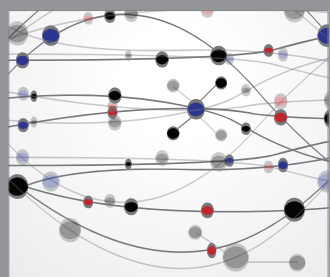

The Scientific World Journal
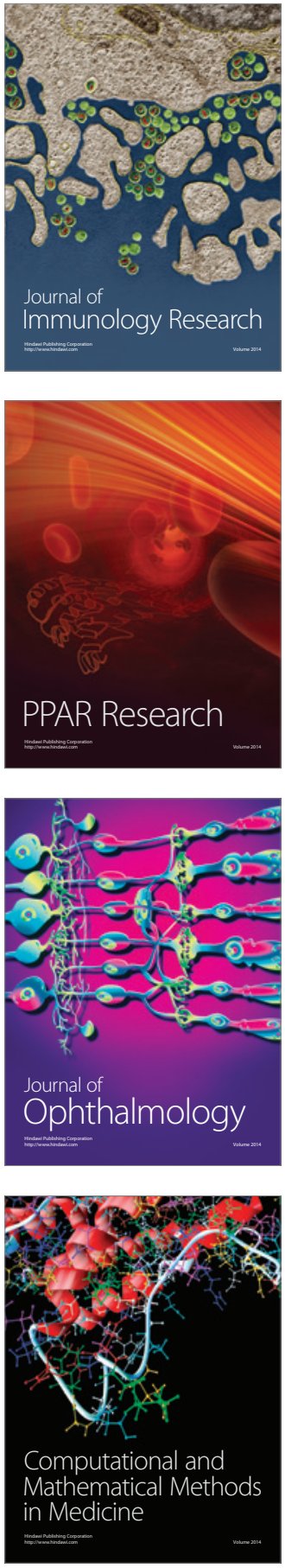

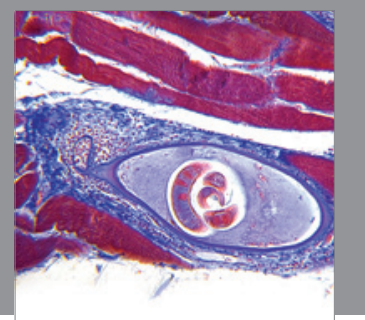

Gastroenterology

Research and Practice
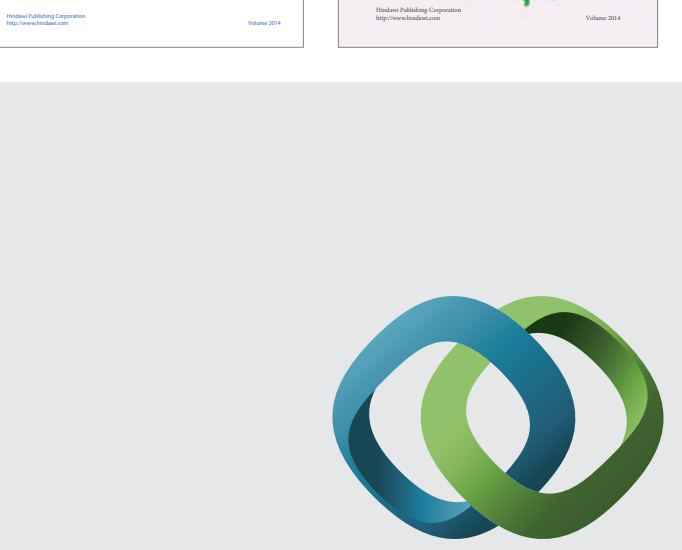

\section{Hindawi}

Submit your manuscripts at

http://www.hindawi.com
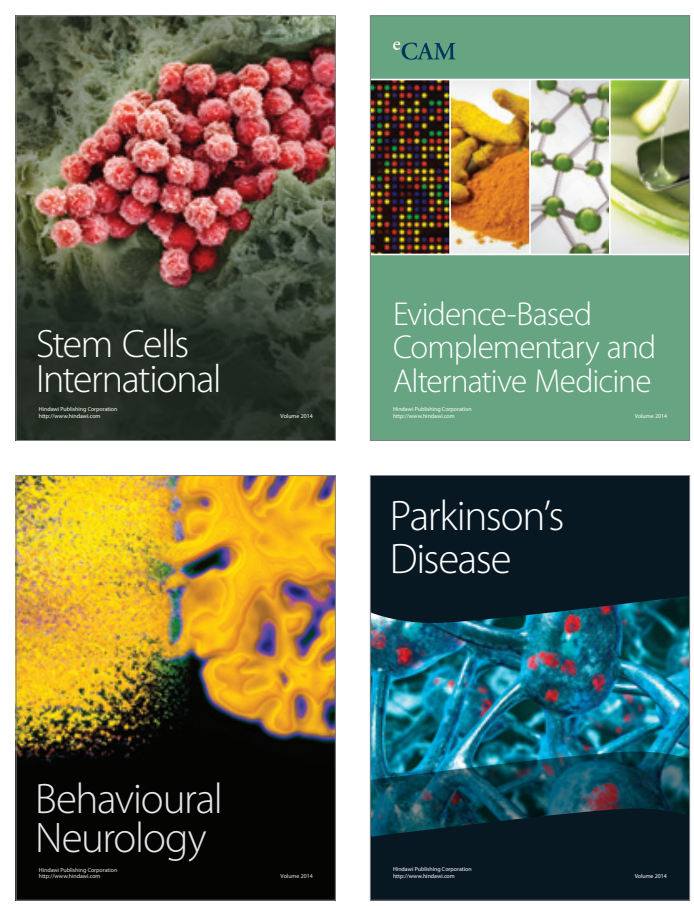

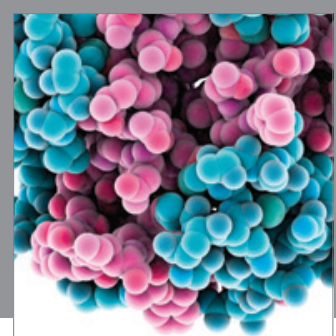

Journal of
Diabetes Research

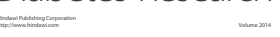

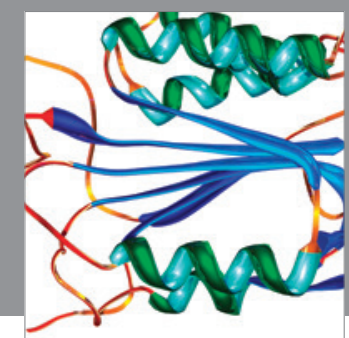

Disease Markers
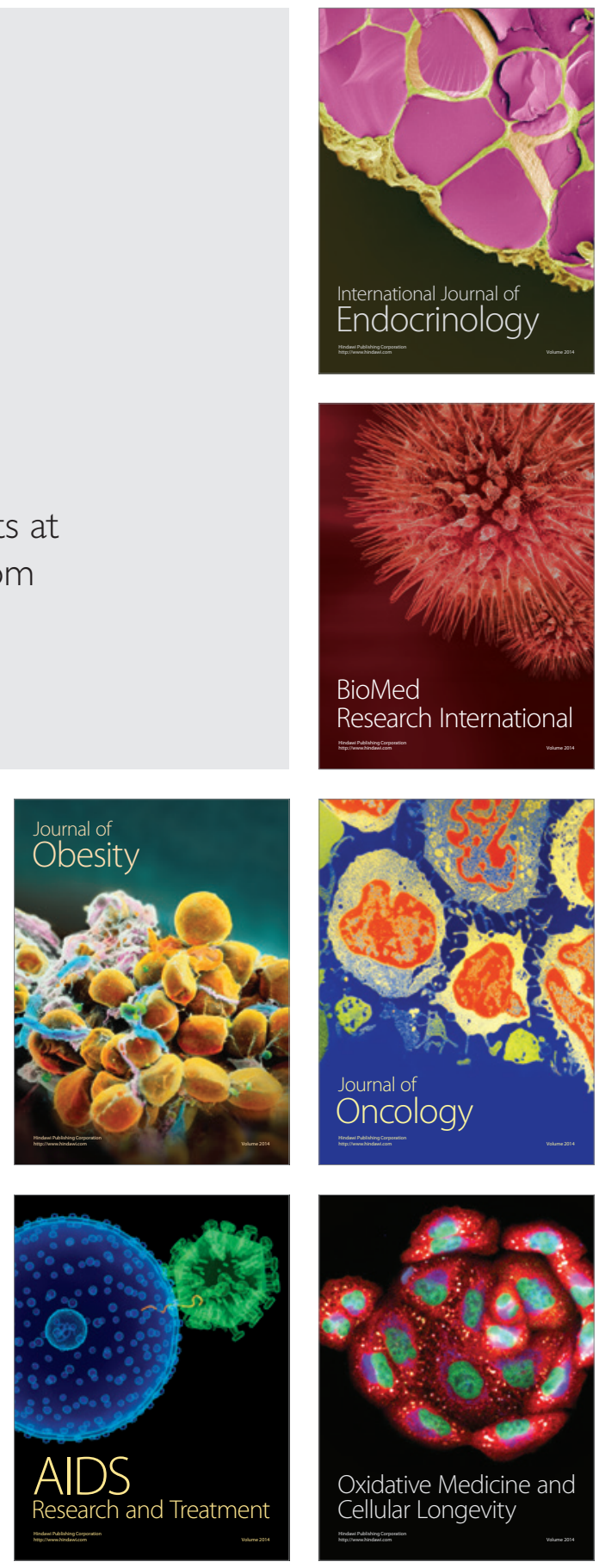